\title{
Referential activity, dissociation, psychopathology and psychotherapy
}

\author{
Raffaella Perrella, Nadia Del Villano, Giorgio Caviglia \\ Department of Psychology, Second University of Naples, Caserta, Italy
}

\begin{abstract}
Within the framework of the research on process in psychotherapy, Wilma Bucci has initiated an attempt to reformulate and widen metapsychological concepts as well as the scope of psychoanalysis towards the contents of neuroscience and cognitive science. Such intent has contributed in developing the multiple code theory: a theoretical-empirical basis for the development of a research method on the evaluation of referential activity - measure of the therapy's global progress through the transcriptions of the sessions. The method ranks in the course of the research on process and stems, as many empirical methods of therapeutic change evaluation, from the crisis of Freud's metapsychology, documented by the various controversies on the theory of the mind at the basis of psychoanalytic practice. From such controversies, it was acknowledged that psychoanalysis requires a modern theory explaining the fundamentals of clinical practice. Bucci's theory represents a development of Freud's differentiation of primary and secondary process: two distinct modes of thought that cannot be proposed in modern reality, since their validity has not been proven yet. Bucci proposes, instead, a three-fold thought and memory organization including verbal symbolic, non-verbal symbolic and sub-symbolic modes of thought. The integration of such modes should accompany the individual in every daily situation, by appearing in his/her language; an example, within psychotherapy, is the patient's free associations. Following traumatic situations, the three systems of thought may not function in an integrated manner: in this sense, psychotherapy may represent the instrument to restore the combined functioning of the three systems.
\end{abstract}

Key words: Referential activity; Dissociation; Trauma; Psychotherapy.

\section{Introduction}

In research on psychotherapy process (Dazzi, 2006), an attempt has begun in reformulating and expanding

Correspondence: Perrella Raffaella, Department of Psychology, Second University of Naples, viale Ellittico 31, 81100 Caserta, Italy.

Tel/Fax: +39.0823.275338.

E-mail: raffaella.perrella@unina2.it

Contributions: RP, NDV and GC developed the study concept. All authors contributed to this work design, drafted the manuscript, and provided critical revisions. All authors approved the final version of the manuscript for submission.

Funding: no funding was provided for this study.

Citation: Perrella, R., Del Villano, N., \& Caviglia, G. (2016). Referential activity, dissociation, psychopathology and psychotherapy. Research in Psychotherapy: Psychopathology, Process and Outcome, 19(2), 165-171. doi: 10.4081/ripppo.2016.243

Received for publication: 23 October 2016.

Revision received: 6 November 2016.

Accepted for publication: 7 November 2016.

This work is licensed under a Creative Commons Attribution NonCommercial 4.0 License (CC BY-NC 4.0).

CCopyright R. Perrella et al., 2016

Licensee PAGEPress, Italy

Research in Psychotherapy:

Psychopathology, Process and Outcome 2016; 19:165-171

doi:10.4081/ripppo.2016.243 meta-psychological and psychoanalytic concepts, both towards neuroscience (Chiesa, 2010) and cognitive science (Horowitz, 1998). One of the main representatives of this project is Wilma Bucci, psychoanalyst, professor at Adelphi University in New York and Scientific Director of the Glass Psychoanalytic Institute of Basic Research in New York, especially known for her multiple code theory. A key impetus to this innovation was given by the crisis of Freudian metapsychology, as documented by the many disputes on the model of the mind underlying the psychoanalytic theory, through which it was understood that psychoanalysis needed a more modern - and empirically founded - theory of mental functioning (Amadei, Cavanna, \& Zavattini, 2015).

Other factors that have contributed to the rise of new psychodynamic theoretical perspectives and, in particular, to the elaboration of Bucci's model, were: i) empirical studies in neuroscience and cognitive science of psychological constructs, such as memory, language, attention, perception; ii) the development of multicomponent models of basic and superior cognitive functions.

Although the psychoanalytic method is considered by many the most coherent and intellectually more satisfactory conception of the mind (Kandel, 2005), it is recognized by many areas of science the need to expand and verify assumptions and key concepts of the method itself, in the light of the new discoveries in other scientific disciplines, like mirror neurons -- specific cells that fire both when the an$\mathrm{imal} /$ human acts and when the animal/human observes the same action, aimed at a purpose, performed by another (Rizzolatti \& Craighero, 2004; Iacoboni, 2008). 
For example, the discovery of mirror neurons, can, in some way, explain some psychoanalytic concepts, like unconscious communication between therapist and patient: these may, unconsciously and with continuity, gather the other's stimuli by activating shared neural patterns. Similarly, we can imagine the same phenomenon to be activated in projective identification. In summary, studies on mirror functioning provide a neurobiological framework to some psychoanalytic concepts and to psychological understanding of the emotional patterns' development, which will be discussed in the following paragraphs (Moccia \& Solano, 2009; Gallese \& Sinigaglia, 2011).

\section{Metapsychology and empirical research}

Psychoanalysis has long been considered the most important psychotherapeutic approach, able to influence each following orientation. The key principles of psychoanalysis include: specific techniques; a psychological model of development; metapsychology, a set of speculative assumptions about the mind's nature, structure and functioning; a specific setting. Revisions on Freud's metapsychology were carried out from the 30 's, and were mainly focused on a major weighting of external reality in the development of the Self, combining Freudian theory and empirical methods, and adapting the theory to new scientific evidence (De Coro \& Ortu, 2010).

The concept that was more in jeopardy, was the drive theory, that implied a closed energy system, with a linear trend, which could not explain thoroughly a dynamic, open, multilinear system as it is, in fact, the human one. The crisis of classical metapsychology, with the development of different theoretical models, opened a debate on the traditional psychoanalytic method, pushing psychoanalysts to question both the status of clinical data and the hypotheses related to therapeutic change factors.

This has led to question whether the Freudian metapsychology is dead or alive, or - even more radically - if psychoanalysis requires an explanatory theory of its clinical practice (Caviglia, Perrella, Sapuppo \& Del Villano, 2010; Ammaniti \& Gallese, 2014).

A successful attempt to reformulate the Freudian metapsychological building was made by Wilma Bucci, who created a method to evaluate referential activity (RA) taking into account converging evidence produced both by clinical and experimental trials, and merging data from psychoanalytic trials, cognitive psychology and neuroscience.

\section{The multiple code theory}

The multiple code theory is defined by the author herself as a psychological theory of emotional intelligence and processing of emotional information, regarding the interactions between different processes and the nature of sensory, motor, somatic, cognitive and language representations (Bucci, 1997; De Coro \& Caviglia, 2000).

This theory is considered an improvement of the Freudian differentiation between primary and secondary process. According to Freud's theory, the primary process - the unconscious mode - is associated to non-verbal functions, while the secondary process - modes of conscious thought - is associated to verbal functions. In fact, the organization of thought and memory is much more complex. Unconscious or implicit thinking can be both verbal and nonverbal, symbolic and sub-symbolic. The contents of implicit, non-verbal, or sub-symbolic thought can well include complex, even mathematical concepts, in addition to satisfaction of desire, in a psychoanalytic point of view. The forms of implicit and nonverbal thinking are realized in normal adult mental life, both when awake and asleep. The explicit or conscious verbal thinking is wide both from a functional point of view and regarding its contents (Bucci, 1997).

In light of this, a two-fold system does not seem sufficient to explain the observed distinctions in the methods of information processing.

Instead of the Freudian duality, the multiple code theory incorporates three main types of information processing systems: non-verbal sub-symbolic elaboration, and the verbal and nonverbal symbolic systems. The distinction between sub-symbolic processes and non-verbal symbolic representations is not covered in the Freudian model: they both are enclosed in the big melting pot of the primary process.

The notion of sub-symbolic mode refers generally to an intuitive and implicit elaboration that sometimes occurs without intention or attention.

The sub-symbolic, nonverbal elaboration concerns all stimuli processed in parallel (as hitting a baseball, distinguishing the taste of a wine from another). This mode of processing is typical of the sensory and kinesthetic components of emotions.

The symbolic, nonverbal elaboration is more prone to emotional control, and depends on explicit parameters; it regards mental images that, although present in the memory, cannot be translated into words.

The symbolic, verbal mode, at last, is a code invented by humans to control and direct themselves, communicate and relate, transmit and preserve the culture; language is majorly subjected to this deliberate control system. Although independent, the three elaboration systems are constantly connected to each other through referential connections, that enable a coordinated functioning. Figure 1 is an attempt to represent figuratively this model.

The multiple code theory replaces the Freudian drive construct with the concepts of emotion schema and emotion information processing, able to explain the arousal and the regulation of human behavior, as well as its organization. Although the notion of emotion schema is a hypothetical construct as the drive, it differs from the lat- 
ter because it is consistent with a general psychological model, subjected to empirical testing. The emotion schemas are active, dynamic, and process new information in an interactive way, determining our perception of experience and constantly modifying themselves with new inputs. Following this model, we can assume that clinical symptoms represent a dissociation of the emotion schemas and a malfunction of the referential process that regulates communication between the three levels. Symptoms and behaviors function as discrete symbolic entities, even if not yet integrated: they can be viewed as steps on the way to a more adequate symbolization, and not only as regression or resistance. This concept is closer to the deficit theories rather than the conflict ones; or to theories on lack of mentalization (Perrella, Semerari \& Caviglia, 2013), rather than to those following an impulse/defense model. As for treatment, the ultimate goal of psychoanalytic therapy was intended as replacing a system with another (like making the unconscious conscious), whereas the multiple code theory considers therapy as reconstruction of dissociated emotion schemas.

In the reformulation of metapsychological concepts and the construction of a new psychoanalytic psychology, some concepts will remain, others will be redefined, while others will be abandoned (Bucci, 1997). According to Bucci, for example, defense mechanisms represent a broad category that includes dissociation of referential connections and dysfunctional strategies. Consider, for example, the case of phobia, with its repression defenses and displacement of symbolic material. Repression, fundamental defense mechanism in Freudian theory, is redefined, in terms of the multiple code theory, as dissociation of referential connections. This can occur between verbal symbolic processes, between words and images (both symbolic), between images and elements of the sub-symbolic process.

As stated above, some psychoanalytic concepts have not been incorporated into the multiple code theory, but rather abandoned or completed. In fact, moving away

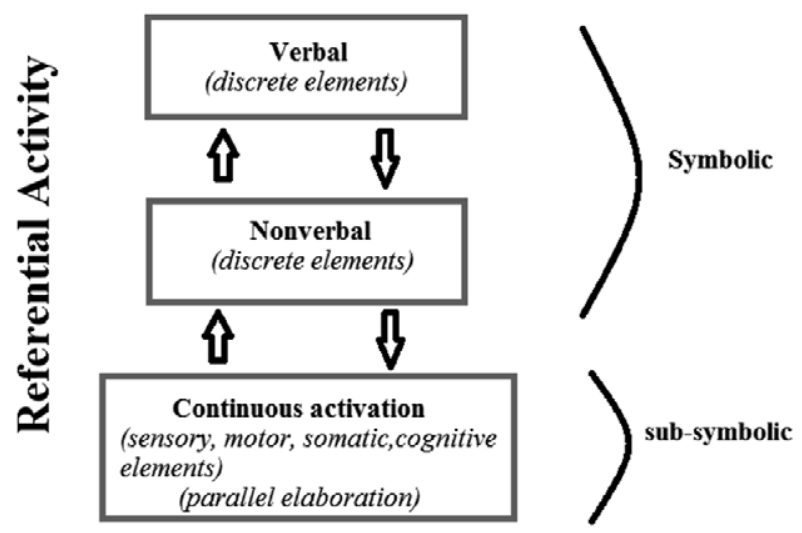

Figure 1. Links of the referential activity. from the two-fold Freudian model towards a multi-class structure, Bucci's theory elaborates the distinction between primary and secondary process. In the multiple code theory, sub-symbolic mental representations have the characteristics both of the primary and the secondary process; they are not verbal, but are organized, accessible to awareness and reality-oriented. Similarly, symbolic language can be associated with forms of regressed thought, dominated by contents regarding satisfaction of desires and processed outside of awareness.

The purpose of Bucci's model is not to replace a complete (yet closed) theoretical system with another, but to provide a solid foundation for empirical research, creating a theoretical building that can be subject to scientific verification. Thanks to the multiple code theory, in fact, an empirical method that can measure change in psychotherapy was developed. This method is based on the evaluation of the construct of RA (Bucci, 1997; De Coro \& Caviglia, 2000; De Coro et al., 2004), which allows to reveal possible associations or dissociations between the three elaboration systems within the various emotion schemas, reactivated during therapy (Caviglia \& Del Villano, 2010).

\section{Free associations and referential cycle}

Through the evaluation of RA, a particular structure within the psychoanalytic session has been highlighted, the referential cycle, which would mark the proper functioning of the therapy. The cycle or referential process, in the interpersonal context of therapy, offers the basic model for the emotional transformation of personal experience (with its dominant sub-symbolic components) into the verbal code, by which the experience can be shared. The process works in different ways, depending on the patient and the degree to which his object representations have stabilized in the early stages of his life, as well as the degree to which dissociation occurred between nonverbal sub-symbolic processes and symbolic ones. The steps followed by the exploration in free associations correspond to those of the referential process: sub-symbolic activation (preparation and incubation), symbolization and illumination (images and words), and reflection and verification.

In the first phase, the patient's work is closely linked to his ability to access sub-symbolic experience and be able to use it, so the treatment should be aimed at developing this capacity. To be able to connect the material in treatment, it is necessary to be willing to talk without knowing what is the real subject, to walk away from one direction or conscious goal, in order to make sure that the sub-symbolic processes direct the research: in this regard, free associations are the most suitable method. Within the treatment, the connection to the sub-symbolic experience proceeds interactively with the development of the relationship, of the connection to new sub-symbolic objects and to the psychotherapist. During a session the focus is on new components of a problematic emotion schema, de- 
termined by the patient's life events or by the treatment. The schema can operate in a dissociated form, and the activation may include sub-symbolic components that emerge without connections to specific objects, or with displaced objects. The emotional core remains not verbalized, inexpressible, painful and inexplicable, given its sub-symbolic form.

The second phase corresponds to the connection process of sub-symbolic components of emotion schemas to images and then words. This stage is divided into the following two sub-phases: construction of a prototypical mental representation and description of images and events.

The first system (construction of a prototypical mental representation) in which the conversion from sub-symbolic to the symbolic form takes place is the nonverbal. The continuous variation of the stimulus, which is part of the sub-symbolic flow, is divided into classes of representation that lead to the production of prototypical images. The patient thinks about a seemingly irrelevant event, memory, image, or dream, and is not aware of the reason why it came to his mind or why it is related to his current experience.

In the description of images and events the patient continues to speak, even though he is not able yet to recognize the emotional significance of images and episodes that emerge. He proceeds with the representations' description, and this reveals his emotion schema in its current version, recalled from memory and expressed in the context of the present situation. During the description, the vicious circle (that prevents the connection between sub-symbolic elements and words) may be broken to start the reorganization of the schema, thereby entering the phase of enlightenment. The psychotherapist's attentive and benevolent presence and the possibility/need to tell, explain and understand (hence referential activity) necessarily lead to an increase in mentalization (Allen \& Fonagy, 2006). The construction of a new schema in treatment is, in some ways, similar to the initial construction in the early stages of development (Del Villano, Perrella, Bisogno \& Caviglia, 2014); the patient, however, now has an adult cognitive structure, his existential situation is different, and the relationship is different from the previous ones. This new relationship gives him the opportunity to internalize a more benevolent and less distorting mental container and to develop new patterns of self-nurturing and self-regulation. The analyst listens, processes the patient's communication with his own emotion schemas, and responds, providing new emotional experience and, in some cases, new categories through interpretation. If the process is successful, the patient is able to use new ideas, information and experiences to develop new categories, new dimensions and to symbolize aspects of the previously dissociated emotion schemas.

In the third phase (reflection and verification), the patient reflects with the analyst on produced images and stories. At this point, the analyst may lead him. Tools as logic differentiation and generalization are intentionally used. The connection of displaced objects to the activated mnemonic scheme can be recognized, as well as differences in the activation situations (Bucci, 1997).

\section{Adaptive functioning vs emergence of psychopathology}

The multiple code theory provides an explanation of emotional information processing using the architecture of three symbolic and sub-symbolic systems. These systems are connected (Figure 1) by an integrative process, RA, a continuous activity that links specific experiences to specific verbal forms. This connection process is an essential human function, operating continuously, required for adaptive functioning. But the formulations of the $m u l-$ tiple code theory can also be extended to the understanding of psychopathology. In particular, one of the situations in which the connection process is interrupted, and consequently alters the individual normal functioning, is the occurrence of a traumatic event.

The multiple code theory explains psychopathology as the disconnection of the three processing systems of information and the dissociation of emotion schemas. These are the basis for the organization of the Self, define our personality and determine our objectives and our choices during lifetime. Any emotion schema may function adaptively or maladaptively, with different features and functioning. Adaptive functioning will be tied to the integrated operation of the components of the emotional scheme (Moccia \& Solano, 2009). Only thanks to integrated schemas new information may be classified or reclassified as a support or interference to the regulatory functions of life, allowing the individual to adapt to different situations. The dissociative or maladaptive functioning of the schema, however, can occur at multiple levels by blocking the entry of new information and possibly the elicitation of appropriate/functional responses.

A first level of dissociation is the failure of the connection between body experience and symbolic representation: the individual is conscious of the emotion, but does not recognize it as an event of the Self. This type of functional dissociation may be associated with traumatic events, abuse, severe disorders; this level of dissociation between schemas may be what explains somatoform phenomena. A second dissociative level concerns the activation of nuclear consciousness without the activation of extended consciousness. In this way, someone can be conscious of an object or event in the present, without linking it with autobiographical memory. The feeling is felt-heard as an event of one's self, but its significance is not recognized, identified and distinguished; so, the feeling is recognized but lacks its emotional significance.

A final and more complex level of dissociation involves the integration of some elements of the extended consciousness, within the scheme, while others are 
blocked or warded off: some components of emotional significance are accessible, while others are not. These last two levels of dissociation regard many dissociative symptoms seen in clinical practice.

These elements are summarized in Figure 2.

\section{Adaptive functioning vs psychopathology}

Dissociation can occur because of an irregular processing of different experiences. It may occur that somatic and sensory components of some lived events - being developed mostly through pre-symbolic elements - cannot to be symbolized with a conscious and verbal meaning and, therefore, experienced as overwhelming or potentially threatening for survival. Somatic and sensory components of traumatic events, as adults, or early experiences of abuse or childhood relational mismatch, are the prototypical experiences of this type of difficulty (Caretti \& Craparo, 2008).

The sub-symbolic processes work with immediacy and effectiveness as long as they're adequate; when they are no longer, the sub-symbolic processor may not be able to operate the elaboration and necessary containment. The change of emotion schemas presents even more difficulty than cognitive and sensory-motor ones. Each element of the emotion schema - a word, an image, an action, a smell - can activate other elements; when the schema is activated by any of its elements, the affective core and the behavioral response associated to it will be activated. For negative and conflicting schemas, sensory and visceral el- ements will probably be associated with suffering (Bucci, 1997). Facing a dissociated complex (consisting in memories, fears, emotions), the individual, attempting to come to terms with the dissociated experience, can perform various compensating mechanisms.

An example is given by the desymbolization mechanism, through which the subject tries to remove or eliminate the painful symbol. Thereby the sub-symbolic affective core is retained, but disconnected from the symbolic level: the pathological schema will continue to reactivate constantly in situations similar to that in which it was recorded. A typical clinical example could be a panic attack.

With resymbolization, instead, the subject gives a new meaning to a painful schema, performing a distortion of its real meaning. Despite being a pathological compensatory mechanism, resymbolization still implies an attempt to symbolization; in this case, a clinical example could be a phobia, or obsessive symptoms. This process can be useful in psychotherapy to connect the original elements through free associations. Within a therapeutic process, the change in an emotion scheme involves: the activation, during the session, of bodily sub-symbolic and sensory experience of the affective core, associated with the events taking place in the therapeutic relationship and past events; the conduction to changes in the emotional significance of the somatic and imaginary experiences; the modulation of the bodily and emotional responses.

So, the change of an emotion schema requires a simultaneous activation of bodily representations, present images and representations of the past. In terms of the

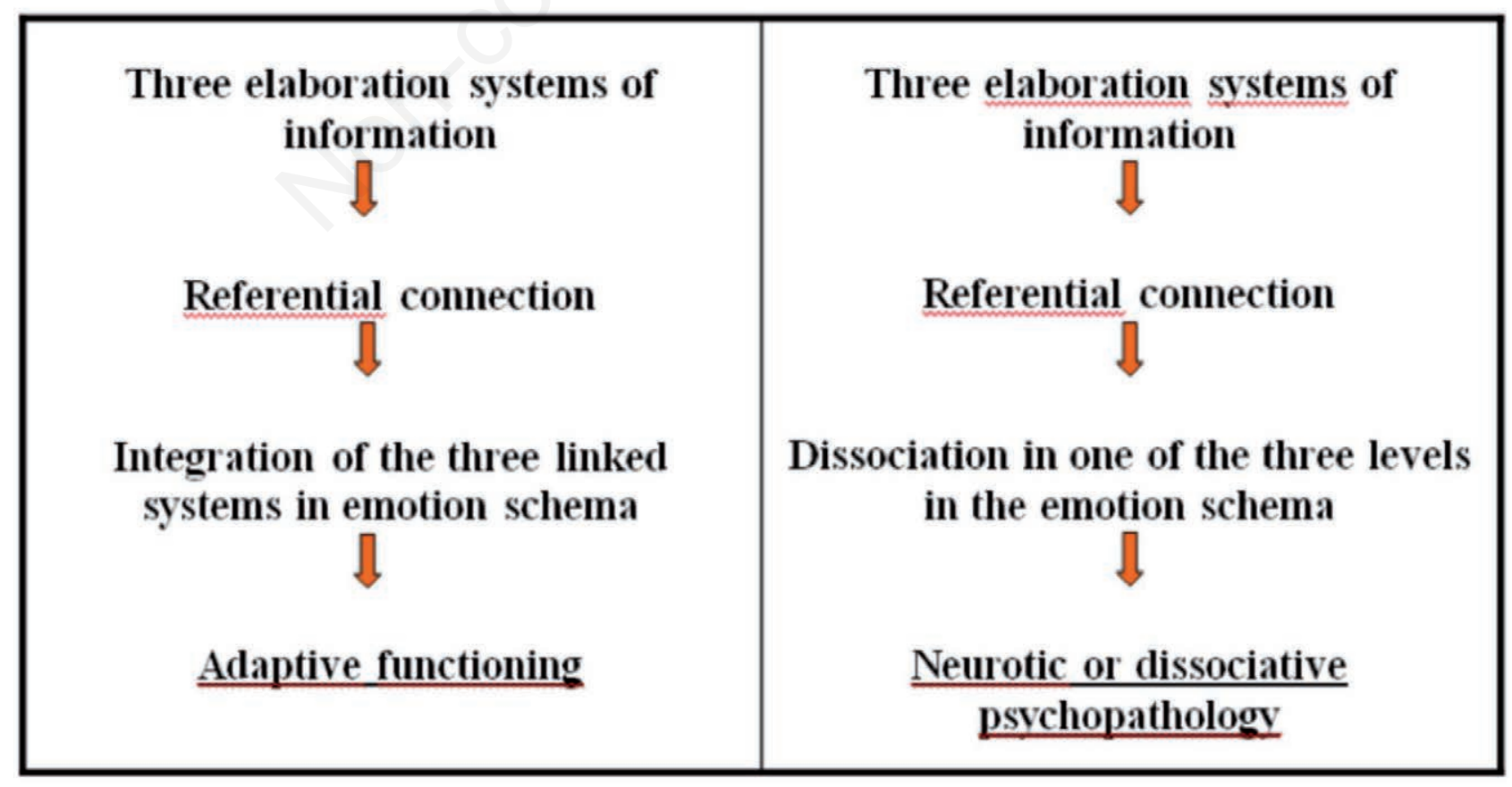

Figure 2. Integration and dissociation. 
multiple code theory all this emerges as an operation of the referential process.

The formulations described above mark further elements of renewal in the Freudian metapsychology, especially on symptoms and defenses. Wilma Bucci, in fact, does not consider the symptom as a substitute discharge, as the symptom will function as a discrete symbolic entity, in the absence of a more appropriate form of symbolization; moreover, symptoms are not only an expression of resistance or regression. Transforming the symptoms verbally, in a referential dialogue (comprehensible to ourselves and others) within the therapy, the individual can begin to symbolize and to reconnect them with dissociated schemas, therefore starting the referential cycle. Another innovative point of this theory lies in the new conceptualization of the defenses: defense mechanisms can be considered as forms of disconnection or dissociation within multiple verbal and non-verbal channels.

\section{Clinical applications and future directions}

One of the merits of the multiple code model consists in having created a theoretical formulation that integrates purely psychoanalytic aspects and ideas from modern cognitive and neurobiological theories. These aspects allow to go beyond the Freudian metapsychological apparatus limits. In fact, Bucci has been able to create an empirical assessment method (see below) on a solid theoretical basis that re-actualizes Freudian metapsychological concepts and clinical insights in the light of developments of modern cognitive science, capable to explain the conscious and the unconscious phenomena. Moreover, the connection with neuroscience adds a biological basis to explain the activated mental functions (Solms \& Bucci, 2000; Moccia \& Solano, 2009).

The Weighted Referential Activity Dictionary (WRAD) is a computerized dictionary based on an empirically derived model of judges' ratings of RA (Bucci \& Maskit, 2006): it includes 697 words that cover $85 \%$ of the words in the corpus that was used to construct the measure. Each of these has a weight that contributes to a score between 0 and 1 , with a neutral value of .5 . The IWRAD represents its adaptation in Italian language (Mariani, Maskit, Bucci \& De Coro, 2013). Through computerized language measures, as Discourse Attributes Analysis Program by Maskit (2012), text analyses can be performed to compute measures of the referential process, as well as other language measures. This method allows evaluating RA in different clinical and research areas, such in secure $v s$ insecure children's narratives (Pazzagli, Mariani, Crisafulli, \& De Coro, 2012), episodic memory impairment in psychiatric samples (Lewis, Murphy \& Hanakawa, 2009) or even in neurological populations (Nelson \& Polignano, 2009). In this last study, specifically, WRAD has been shown to differentiate participants with Alzheimer's dementia from nonclinical controls, by eliciting personal memories of the day John F. Kennedy was assassinated in 1963 and the attacks of September 11, 2001 and subsequently analyzing RA with the DAAP (Nelson \& Polignano, 2009).

\section{Conclusions}

Bucci's method can also be used as a support to the empirical evaluation of clinical change, since it can identify, through language analyses, the variations between emotional-affective, deep experiences and experiences reported only in a repetitive, rationalized and frozen way. Also, it is possible to identify specific indicators of a good outcome in psychotherapy, such as the patient's ability to link reflective processes and felt emotions, the occurrence of referential cycles, and the presence of organized and coherent narratives (Lo Verde, Sarracino \& Vigorelli, 2012) by evaluating RA. At this point, one of the aims of psychoanalytic therapy can be redefined as to reintegrate, in the organization of representations of self and others (through the verbalization and the subsequent elaboration), those aspects of emotional experience dissociated from cognitive elaboration. Abandoning the defensive splitting and integrating sub-symbolic and symbolic codes should, in fact, allow a re-elaboration of previously disconnected emotions.

\section{References}

Allen, J.G., \& Fonagy, P. (2006). Mentalization-based treatment. Chichester: John Wiley.

Amadei, G., Cavanna, D., \& Zavattini, G.C. (2015). Psicologia dinamica. Bologna: Il Mulino.

Ammaniti, M. \& Gallese, V. (2014). The birth of intersubjectivity: psychodynamics, neurobiology, and the self. New York: WW Norton \& Company.

Bucci, W. (1997). Psychoanalysis and cognitive science. New York: Guilford Press.

Bucci, W., \& Maskit, B. (2006). A weighted dictionary for referential activity. In J.G. Shanahan, Y. Qu, \& J. Wiebe (Eds.) Computing attitude and affect in text (pp. 49-60). Dordrecht: Springer.

Caretti, V., \& Craparo, G. (2008). Trauma e psicopatologia. Un approccio evolutivo-relazionale [Trauma and psychopathology. An evolutive and relational approach]. Rome: Astrolabio.

Caviglia, G., \& Del Villano, N. (2010). Attività referenziale, dissociazione e psicopatologia [Referential activity, dissociation and psychopathology]. Psichiatria e Psicoterapia, 3, 139-155.

Caviglia, G., Perrella, R., Sapuppo, W., \& Del Villano, N. (2010). La ricerca in psicoterapia: il contributo del Gruppo di lavoro della Cattedra di Psicologia Dinamica della Seconda Università di Napoli [Research in psychotherapy: a contribution by the Board of the Department of Dynamic Psychology, Second University of Naples]. Research in Psychotherapy, 2(13), 32-52.

Chiesa, M. (2010). Research and psychoanalysis. Still time to bridge the great divide? Psychoanalytic Psychology, 7(2), 94-114. 
Dazzi, N. (2006). Il dibattito contemporaneo sulla ricerca in psicoterapia [Contemporary debate upon research in psychotherapy]. In N. Dazzi, V. Lingiardi, \& A. Colli (Eds.) La ricerca in psicoterapia. Modelli e strumenti [Research in psychotherapy. Models and instruments]. Milan: Raffaello Cortina.

De Coro, A., \& Caviglia, G. (2000). La valutazione dell'attività referenziale [Evaluation of referential activity]. Rome: Kappa.

De Coro, A., Mariani, R., Pazzagli, C., Andreassi, S., Ortu, F., \& Caviglia, G. (2004). Il nuovo dizionario computerizzato per la valutazione dell'attività referenziale. Un'applicazione allo studio del caso singolo [New computerized dictionary for evaluating referential activity. An application to the study of a single case]. Sixth National Congress of clinical psychology section of A.I.P., 23-25 October 2004, Aosta, Italy.

De Coro, A., \& Ortu, F. (2010). Psicologia dinamica. I modelli teorici a confronto [Dynamic psychology. A comparison of theoretical models]. Bari: Laterza.

Del Villano, N., Perrella, R., Bisogno, S., \& Caviglia, G. (2014). Working memory abilities, attachment relationships and learning process in children of primary school age: an empirical research. Research in Psychotherapy: Psychopathology, Process and Outcome, 17(1), 21-32.

Gallese, V., \& Sinigaglia, C. (2011). What is so special about embodied simulation? Trends in Cognitive Sciences, 15(11), 512-519.

Horowitz, M.J. (1998). Cognitive psychodynamics. New York: John Wiley.

Iacoboni, M. (2008). Ineuroni specchio [Mirror neurons]. Turin: Boringhieri.

Kandel, E.R. (2005). Psichiatria, psicoanalisi e nuova biologia della mente [Psychiatry, psychoanalysis and new biology of the mind]. Milan: Raffaello Cortina.

Lewis, K., Murphy, S., \& Hanakawa, Y. (2009). Uncovering episodic memory through linguistic measures in schizophrenia. Supplement to the poster presented at the Association for Psychological Science Annual Convention, San Francisco, CA, USA.
Lo Verde, R., Sarracino, D., \& Vigorelli, M. (2012). Therapeutic cycles and referential activity in the analysis of the therapeutic process. Research in Psychotherapy: Psychopathology, Process and Outcome, 15(1), 22-31.

Mariani, R., Maskit, B., Bucci, W., \& De Coro, A. (2013). Linguistic measures of the referential process in psychodynamic treatment: the English and Italian versions. Psychotherapy Research, 23(4), 430-447.

Maskit, B. (2012). The Discourse Attributes Analysis Program (DAAP) (Series 8) [Computer software]. Available from: http://www.thereferentialprocess.org/the-discourse-attributes-analysis-program-daap

Moccia, G., \& Solano, L. (2009). Psicoanalisi e neuroscienze. Risonanze interdisciplinari [Psychoanalysis and neurosciences. Multidisciplinary approaches]. Milan: Franco Angeli.

Nelson, K., \& Polignano, M. (2009). Referential activity in negative episodic 'flashbulb' memories from patients. Poster session at the Association for Psychological Science Annual Convention, San Francisco, CA, USA.

Pazzagli, C., Mariani, R., Crisafulli, V., \& De Coro, A. (2012). Relazione tra attaccamento e misure linguistiche computerizzate dell'attività referenziale: uno studio esplorativo sulle narrative in età evolutiva [Relationship beween attachment and computerized linguistic measures of referential activity: an exploratory study on narration in childhood and adolescence]. Infanzia e adolescenza, 11(2), 102-112.

Perrella, R. Semerari, A., \& Caviglia, G. (2013). Metacognition, borderline pathology and psychotherapeutic change: a single-case study. Research in Psychotherapy: Psychopathology, Process and Outcome, 16(2), 102-108.

Rizzolatti, G., \& Craighero, L. (2004). The mirror neurons system. Annual Review of Neuroscience, 27, 169-192.

Solms, M., \& Bucci W. (2000). Biological and integrative studies on affect. International Journal of Psychoanalysis, 81, 141-144. 